\title{
Gender Transitioning before Puberty?
}

\author{
Thomas D. Steensma $\cdot$ Peggy T. Cohen-Kettenis
}

Published online: 4 March 2011

(C) Springer Science+Business Media, LLC 2011

In the last decade, delaying puberty by means of $\mathrm{GnRH}$ analogs in gender dysphoric adolescents has become an increasingly accepted treatment (Hembree et al., 2009). The induced pubertal delay is meant to give gender dysphoric adolescents time to reflect on their wish for gender reassignment, quietly and without the alarming physical puberty development. During puberty suppression, a complete social transition (change in clothing and hair style, first name, and use of pronouns) is not required. However, most youth who are on puberty delaying hormones appear not to wait with transitioning until they can start crosssex hormone treatment.

A similar trend can be observed in gender variant prepubertal children. For quite some time, gender variant children who came to clinical attention were treated by psychotherapy with the purpose of decreasing cross-gender behavior and identification (Zucker, 2008). More recently, a more gender affirmative approach has been proposed (e.g., Saeger, 2006). This approach may involve complete social transitioning (including a change of first name and pronouns) of children as young as 4 or 5 years of age. Even without contacting clinicians, an increasing number of parents also support young children in their wish to live in the desired gender role on a daily basis. Before the year 2000, 2 (1.7\%) prepubertal boys out of 112 referred children to the Amsterdam gender identity clinic were living completely in the female role. Between 2000 and 2004, 3.3\% (4 out of 121 children; 3 boys and 1 girl) had completely transitioned (clothing, hairstyle, change of name, and use of pronouns) when they were referred, and 19\% (23 out of 121 children; 9 boys and 14 girls) were living in the preferred gender role in clothing style and hairstyle, but did not announce that they wanted a name and pronoun

T. D. Steensma $(\bowtie) \cdot$ P. T. Cohen-Kettenis

Department of Medical Psychology, VU University Medical Center, PO Box 7057, 1007 MB Amsterdam, The Netherlands e-mail: t.steensma@vumc.nl change. Between 2005 and 2009, these percentages increased to $8.9 \%$ ( 16 out of 180 children; 10 boys and 6 girls) and $33.3 \%$ (60 out of 180, 17 boys and 43 girls) respectively.

Such an approach assumes a high persistence of gender dysphoria or gender identity disorder (GID) after puberty. However, follow-up studies show that the persistence rate of GID is about $15.8 \%$ (39 out of the 246 children who were reported on in the literature) (for an overview, see Steensma, Biemond, de Boer, \& Cohen-Kettenis, 2011), and that a more likely psychosexual outcome in adulthood is a homosexual sexual orientation without gender dysphoria (Wallien \& Cohen-Kettenis, 2008).

We wondered what would happen to children who transitioned in childhood, but discover at an older age that they preferred to live in the gender role of their natal sex again. Recently, we conducted a qualitative study among older adolescents who had been gender dysphoric in childhood (Steensma et al., 2011). Some of these children appeared to be persisters and they applied for gender reassignment when entering puberty. Others appeared to be desisters and were only interviewed for the follow-up study. In the desisting group, two girls, who had transitioned when they were in elementary school, reported that they had been struggling with the desire to return to their original gender role, once they realized that they no longer wanted to live in the "other" gender role. Fear of teasing and shame to admit that they had been "wrong" resulted in a prolonged period of distress. Only when they started high school did they dare to make the change back.

Although gender affirmative treatment, including a complete social transition, may be beneficial for children who will turn out to be persisters, clinicians and caregivers should realize that prediction of an individual child's psychosexual outcome is very difficult in young children. It is conceivable that the drawbacks of having to wait until early adolescence (but with support in coping with the gender variance until that phase) may be less serious than having to make a social transition twice. 
Because the chances are high that the gender dysphoria will disappear by early adolescence, it seems advisable to be very careful when taking steps that are difficult to reverse.

\section{References}

Hembree, W. C., Cohen-Kettenis, P. T., Delemarre-van de Waal, H. A., Gooren, L. J., Meyer, W. J., Spack, N. P., et al. (2009). Endocrine treatment of transsexual persons: An Endocrine Society practice guideline. Journal of Clinical Endocrinology and Metabolism, 94 , 3132-3154.
Saeger, K. (2006). Finding our way: Guiding a young transgender child. Journal of GLBT Family Studies, 2, 207-245.

Steensma, T. D., Biemond, R., de Boer, F., \& Cohen-Kettenis, P. T. (2011). Desisting and persisting gender dysphoria after childhood: A qualitative follow-up study. Clinical Child Psychology and Psychiatry. doi:10.1177/1359104510378303.

Wallien, M. S., \& Cohen-Kettenis, P. T. (2008). Psychosexual outcome of gender-dysphoric children. Journal of the American Academy of Child and Adolescent Psychiatry, 47, 1413-1423.

Zucker, K. J. (2008). Children with gender identity disorder: Is there a best practice? Neuropsychiatrie de l'Enafance et de l'Adolescence, $56,358-364$. 